\title{
How Dopants and Defects Affect the properties of Thermoelectric $\mathrm{Ca}_{3} \mathrm{Co}_{4} \mathrm{O}_{9}$
}

Q. Qiao, ${ }^{1}$ A. Gulec, ${ }^{1}$ T. Paulauskas, ${ }^{1}$ S. Kolesnik, ${ }^{2}$ B. Dabrowski, ${ }^{2}$ M. Ozdemir, ${ }^{3}$ C. Boyraz, ${ }^{3}$ D. Mazumdar, ${ }^{3}$ A. Gupta, ${ }^{3}$ and Robert F. Klie ${ }^{1}$

${ }^{1}$ Department of Physics, University of Illinois at Chicago, Chicago, IL

${ }^{2}$ Department of Physics, Northern Illinois University, DeKalb, IL

${ }^{3}$ Center of Materials for Information Technology, University of Alabama, Tuscaloosa, AL

Thermoelectric oxides have attracted increasing attention due to their high thermal power and temperature stability.[1] In particular, $\mathrm{Ca}_{3} \mathrm{Co}_{4} \mathrm{O}_{9}(\mathrm{CCO})$, a misfit layered structure consisting of single layer hole-doped $\mathrm{CoO}_{2}$ sandwiched between insulating $\mathrm{Ca}_{2} \mathrm{CoO}_{3}$ rocksalt layers, exhibits a high Seebeck coefficient at $1000 \mathrm{~K}$. It was suggested that the Seebeck-coefficient can be further increased by growing doped thin films with controlled defects structures.[2] This study combines pulsed layer deposition thin film synthesis of pristine CCO on several oxide substrates, as well as $\mathrm{CCO}$ thin films doped with $\mathrm{Ti}, \mathrm{Bi}$ or $\mathrm{La}$, with aberration-corrected scanning transmission electron microscopy and electron energy loss spectroscopy (EELS) in the JEOL ARM200-CF to examine the effects of interfacial strain and doping on the atomic and electronic structures of CCO. The thermoelectric properties will be measured and correlated to the local changes in the atomic and electronic structures. We will further evaluate the role of $\mathrm{CoO}_{2}$ stacking faults, as well as film thickness on the thermoelectric properties of CCO.

Previously, using atomic-column-resolved EELS and in-situ heating experiments, we have demonstrated that there is significant charge transfer between the Co-ions in the insulating rocksalt layers and the metallic $\mathrm{CoO}_{2}$-layers. In addition, we could show that at high temperature the Co-ion spin-states undergo a transition into a higher spin-state without any structural transition. [3-4]

Figures 1 shows atomic-resolution Z-contrast images of bulk CCO and a $40 \mathrm{~nm}$ thin CCO film on $\mathrm{SrTiO}_{3}$. The thin film exhibits a significant increase in the Seebeck coefficient, and a high density of stacking faults, as well as $a b$-plane rotation. Moreover, the thin films appear unstrained, although the lattice mismatch between the $\mathrm{SrTiO}_{3}(001)$ substrate ad the CCO (001) thin film is $14.3 \%$. Figure 2 shows an atomic-column resolved EELS spectrum image of the area marked in Figure 1b). The integrated Co L-edge intensity shows that the stacking faults consist of a double $\mathrm{CoO}_{2}$ layer, while the relative $\mathrm{O}$ K-edge pre-peak exhibits its highest intensity on the O-atomic columns of teh $\mathrm{CoO}_{2}$ layers. The relative $\mathrm{O}$ K-edge pre-peak intensity is significantly increased in the stacking fault (Figure 2c), pointing to a higher mobile hole concentration or Co-ion spin state. The effects of $\mathrm{Ti}$ doping in $\mathrm{CCO}$ are explored in Figure 3, which shows a Z-contrast image of a $\mathrm{Ca}_{3} \mathrm{Co}_{3.8} \mathrm{Ti}_{0.2} \mathrm{O}_{9}$ thin film. It can be clearly seen that Ti does not replace Co but instead sits in the Ca atomic columns, and does therefore not induce any strain or provide additional mobile holes to the $\mathrm{CoO}^{2}$ layers.

In our presentation we will discuss how the combination of atomic-resolution Z-contrast imaging, EELS spectrum imaging and transport measurements can determine the effects of structural disorder, strain and charge transfer on the thermoelectric properties of $\mathrm{Ca}_{3} \mathrm{Co}_{4} \mathrm{O}_{9}$-based materials. [5]

References:

[1] A. C. Masset et al., Phys Rev B 62, 166 (2000) 
[2] I. Matsubara et al., Appl. Phys. Lett, 80 (25), 4729-4731 (2002)

[3] G. Yang, Q. Ramasse, and R. F. Klie, Physical Review B 78 (15), 153109 (2008).

[4] G. Yang, Q. Ramasse, and R.F. Klie, Appl. Phys. Lett, 94 (9), 093112 (2009)

[5] This research was supported by a Grant from US Army Research Office under contract number W911NF-10-1-0147 and the National Science Foundation (Grant No. DMR-0846748)
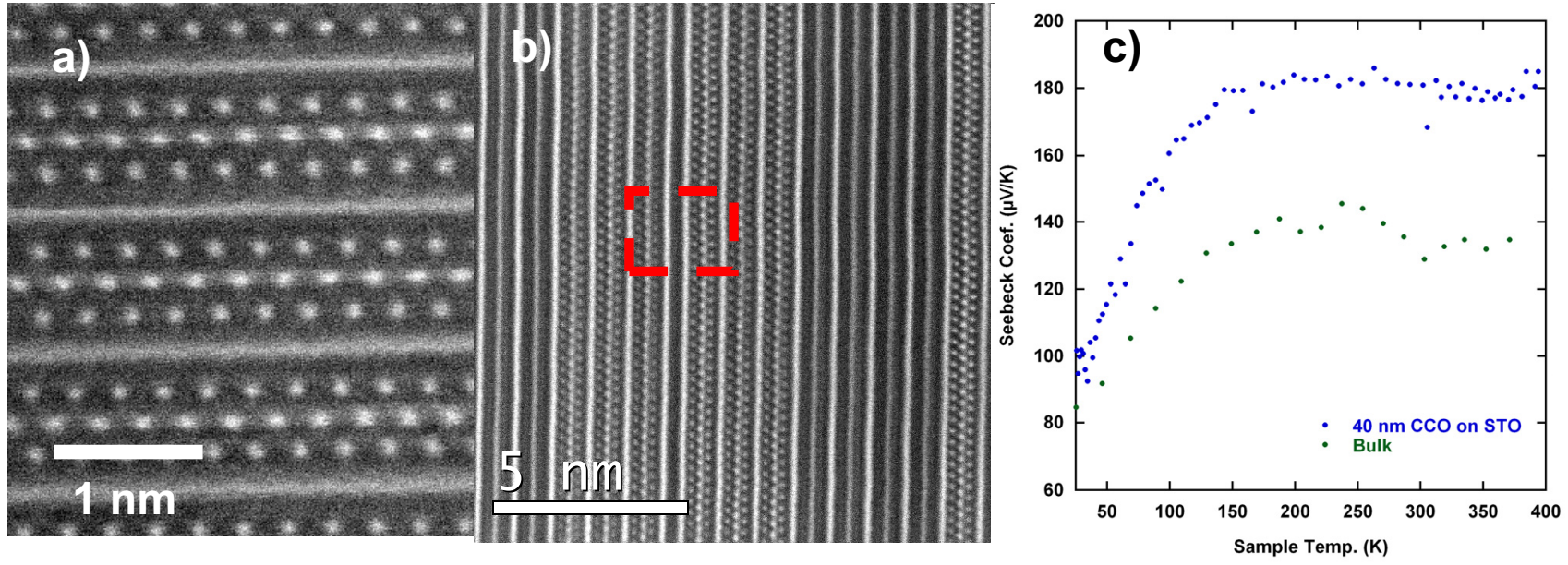

Figure 1: Atomic-resolution Z-contrast image of $\mathrm{Ca}_{3} \mathrm{Co}_{4} \mathrm{O}_{9}$ [110] of a) bulk and b) the $40 \mathrm{~nm}$ thin film on $\mathrm{SrTiO}_{3}$ showing $\mathrm{CoO}_{2}$ stacking fault. c) Seebeck coefficient for both samples.
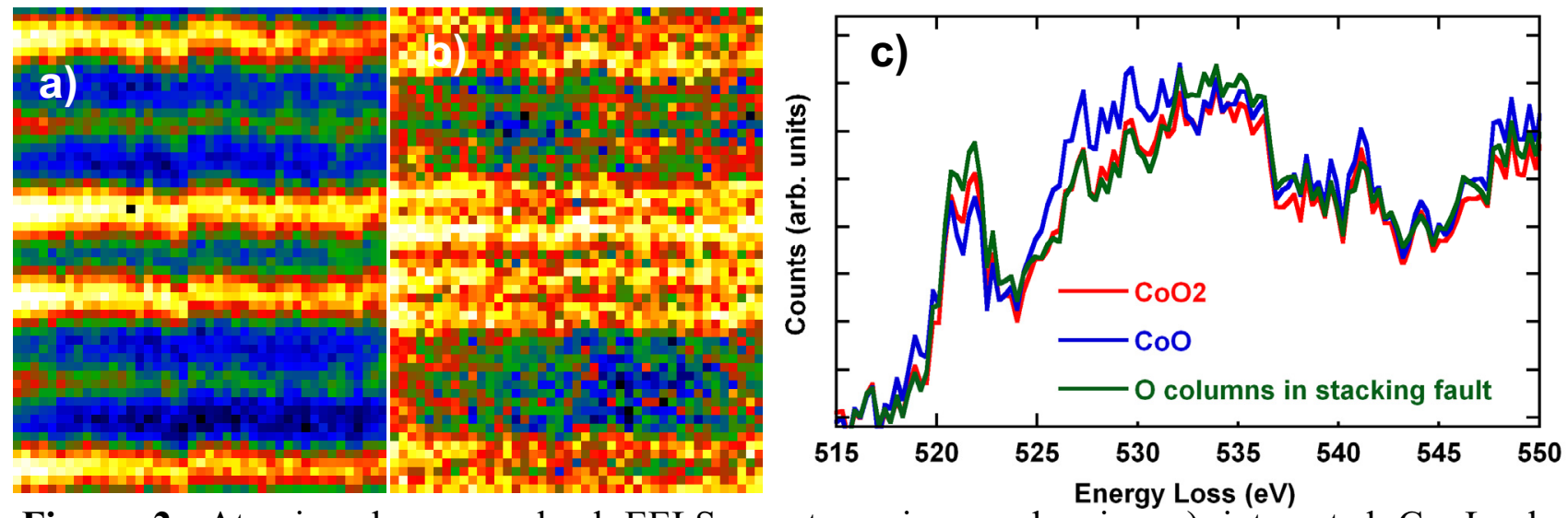

Figure 2: Atomic-column resolved EELS spectrum image showing a) integrated Co L-edge intensity, b) relative $\mathrm{O}$ K-edge pre-peak intensity across $\mathrm{CoO}_{2}$ stacking fault. c) $\mathrm{O}$ K-edge fine structure for the different layers in $\mathrm{CCO}$, showing the increased pre-peak in the $\mathrm{CoO}_{2}$ stacking fault.
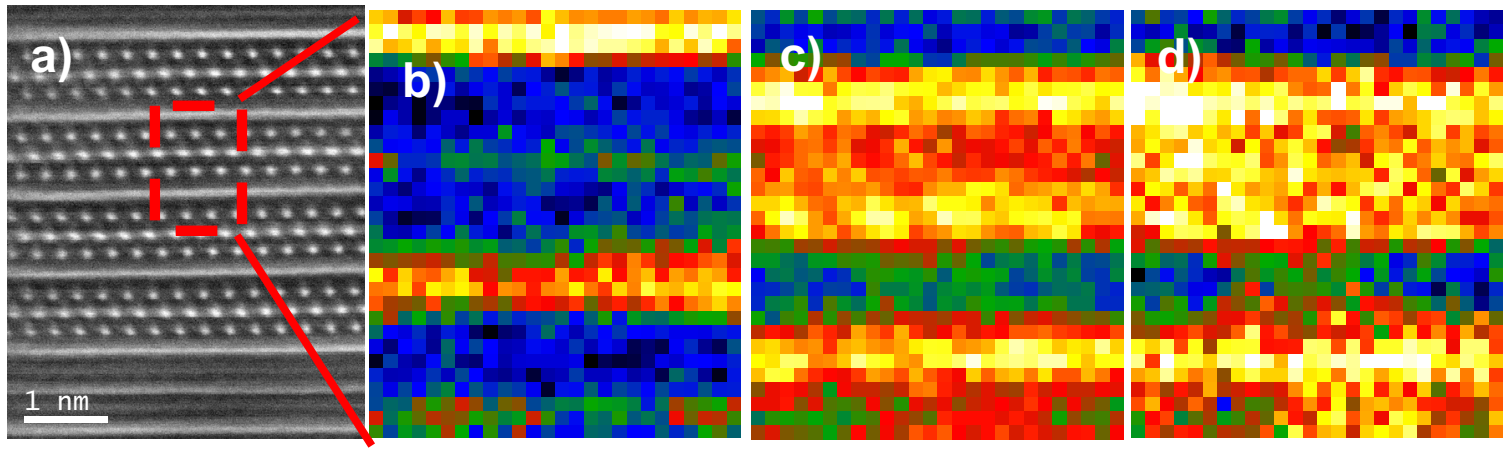

Figure 3: a) Atomic-resolution Z-contrast image of $\mathrm{Ca}_{3} \mathrm{Co}_{3.8} \mathrm{Ti}_{0.2} \mathrm{O}_{9}$ thin film. b-d) Atomic column resolved spectrum image of the b) $\mathrm{Co} \mathrm{c}$ ) $\mathrm{Ca}$ and d) $\mathrm{Ti}$ L-edges showing that the $\mathrm{Ti}$ dopants are replacing $\mathrm{Ca}$ atoms rather than $\mathrm{Co}$. 